\title{
Forward-backward multiplicity correlations in pp collisions in ALICE at 0.9, 2.76 and $7 \mathrm{TeV}$
}

\section{Grigory Feofilov*}

St.Petersburg State University(Russia)

E-mail: feofilovdhiex.phys.spbu.ru

\section{Igor Altsybeev}

St.Petersburg State University(Russia)

Vladimir Vechernin

St.Petersburg State University(Russia)

\section{Sudipan De}

Department of Atomic Energy (India)

\section{Tapan Nayak}

Department of Atomic Energy (India)

\author{
Brijesh Kumar Srivastava \\ Purdue University (United States of America)
}

\section{(for ALICE Collaboration)}

\begin{abstract}
Dependence of the Forward-Backward (FB) charged-particle multiplicity correlations on position and width of separate rapidity windows is investigated in proton-proton (non-single diffractive) collisions at $\sqrt{s}=0.9,2.76$ and $7 \mathrm{TeV}$ with ALICE at the LHC. Two pseudorapidity intervals (the "forward" and the "backward" windows) of the variable width from 0.2 to 0.8 rapidity units were chosen. Strong and non-trivial dependence of the multiplicity correlation coefficient, obtained for the normalized observables, on the width of the pseudorapidity windows is reported. In addition, we observe an increase of the multiplicity correlation strength with the collision energy. Results are discussed and compared to PYTHIA simulations.
\end{abstract}

XXI International Baldin Seminar on High Energy Physics Problems

September 10-15, 2012

JINR, Dubna, Russia

* Speaker. 


\section{Introduction.}

The present study is devoted to the multiplicity backward-forward (FB) correlations in minimum bias $p p$ collisions at $0.9,2.76$ and $7 \mathrm{TeV}$ measured with the ALICE at the Large Hadron Collider (LHC) in line with the physics programme [四]. The investigation of these correlations between various observables in two different, sufficiently separated pseudorapidity intervals (windows0, may be a powerful tool for defining the initial conditions for the formation of the QGP [[]],[[]]. Results could also discriminate the existing MC models based on the combination of the pQCD and non-pertubative phenomenological approaches [䧃].

These backward-forward correlations may include both "short-" and "long-range " components. The first ones could be defined as those relevant to rather narrow pseudorapidity region $\left(\eta_{\text {gap }}<1\right)$, where various energy-conservation short-range effects may dominate (resonance decays, jets, back-to-back correlations, etc.) [42]. "Long-range "correlations are usually assumed to be those extended over a wider range in pseudorapidity, beyond $\eta_{\text {gap }}>1$. However, it will be shown below that scanning in the rapidity space and investigations of the forward-backward correlation strength as a function of the pseudorapidity window size provides valuable information on the short- and long-range components even in the case of limited in pseudorapidity acceptance of $|\eta|<0.8$ of the ALICE tracking detectors.

Forward-backward multiplicity correlations have been studied across a wide range of energies and colliding species, the correlation strength $b_{c o r}$ has been measured in $\mathrm{e}^{+} \mathrm{e}^{-}, p p, p \bar{p}, \pi+p, K+p$

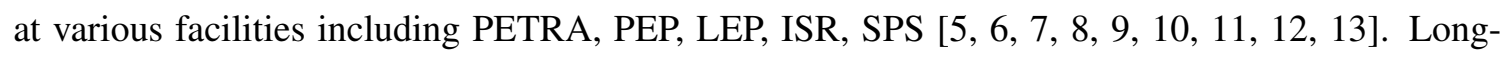
range correlations extended up to 5 units of pseudorapidity were observed in $p \bar{p}$ collisions at 0.3-1.8 $\mathrm{TeV}[155]$. The first detailed study of correlations in $\mathrm{Pb}-\mathrm{Pb}$ collisions using event-by-event data for multiplicity and mean transverse momenta of charged paricles in separated rapidity intervals was performed at the SPS at the laboratory energy $158 \mathrm{AGeV}\left(\sqrt{s_{N N}}=17 \mathrm{GeV}\right)$ [प]]. The measurement of forward-backward multiplicity correlations in nucleus-nucleus collisions has been demonstrated by the STAR experiment at RHIC [18, [9]. These results have generated a great deal of theoretical

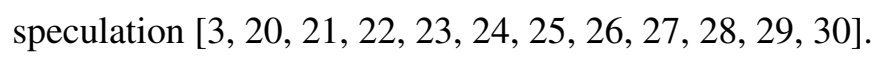

The major physics motivation for the present study is based on the concept of the chromoelectric flux tubes (quark-gluon strings or Pomerons exchanged between the colliding hadrons) the objects stretched in rapidity [B]], that might be formed at the very early stages of hadron-hadron collisions. These color strings may interact[[32] and, in case of sufficiently high density, string fusion may take place producing new strings of higher color[[3]]. This new phenomenon of string fusion could be one of the mechanisms, leading to the QGP formation. It was shown [B4] that the study of backward-forward correlations of particle production in collisions of two nuclei at high energies may allow to distinguish between the string fusion, the clusters formation and decay processes. The long-range correlations between various observables, measured in sufficiently separated rapidity intervals, may appear as the signal relevant to string fusion, while decay of clusters of particles and resonances is marked by increased short range correlations. Recently, similar predictions for the LRC appeared also in the model of Color Glass Condensate and Glasma flux tubes [B]], originating due to the formation of so-called "glasma" flux tubes at the very early collisions stages. However these models (SFM[B4] , and CGC[B5], [B6]) differ in the predictions of behavior of multiplicity correlation strength with the centrality of collision. The presence of strong long- 
range backward-forward multiplicity correlations extended to several units of pseudorapidity, was demonstrated for the MC event generator PYTHIA [ㅍ]]. These correlations were analyzed in comparison to experimental data[ㄷ]] obtained in $p \bar{p}$ collisions at $0.3-1.8 \mathrm{TeV}$ collision energies, and the increased role of the color reconnection processes included in PYTHIA was discussed. In addition, the multiple parton interactions (MPI), considered by PYTHIA, might be also responsible for the fluctuation in the number of color strings, and, therefore, could be one of the potential contributors to the LRC [四]. In all approaches shown above the LRC are produced by the fluctuating number of particle emitting sources (strings), formed in the collision, although the dynamical origin of the sources might be different. Therefore, the detailed experimental studies of the correlation strength and its rapidity structure are crucial for understanding of the physics of the hadron collision process in general.

We are investigating in the present work the backward-forward correlations of charged particles yields (the multiplicity correlations) in $p p$ collisions at the LHC.

The paper is organized as follows. We start from the definition of the forward-backward correlation strength. We describe the procedures of the event and track selection, the efficiency corrections and systematic error estimates and cross-checks. Then we present our experimental results on multiplicity backward-forward correlation measurements done by ALICE in $p p$ collisions at the LHC at $\sqrt{s}=0.9,2.76$ and $7 \mathrm{TeV}$. Scanning in the width and position of the backward and forward windows provides the possibility of detailed study of the rapidity dependence of the correlation strength. Finally, the results are compared to PYTHIA MC event generator calculations and discussed.

\section{Correlation strength}

Two separate pseudorapidity intervals or "windows" (with variable width from 0.2 to 0.8 rapidity units) are defined as "forward" $\left(\delta \eta_{F}\right)$ and "backward" $\left(\delta \eta_{B}\right)$. In the analysis these windows and marked by indices $B$ and $F$ (these indices could be skipped for simplicity in some cases below).

Correlations between the values of multiplicity of charged particles $(n)$ in the given event, measured in the given selected and separated pseudorapidity $\delta \eta$ windows, are investigated as a function of distance (or gap) between the windows. These pseudorapidity windows are symmetrically chosen in the ALICE TPC $\eta$ region $(-0.8,0.8)$. We also study and report here the dependence of the correlation strength on the value of the acceptance $\delta \eta$ of the windows. We have to note here that the additional studies by ALICE of the backward-forward multiplicity correlations for the separated azimuthal $(\delta \phi)$ sectors are also in progress in a similar way in line with [四].

In order to describe these correlations numerically usually the average value $\langle B\rangle_{F}$ of one dynamical variable $B$ in the backward rapidity window $\delta \eta_{B}$ as a function of another dynamical variable $F$ in the forward rapidity window $\delta \eta_{F}$ is used.

Here $\langle\ldots\rangle_{F}$ denotes averaging over the events at a fixed value of the variable $F$ in the forward rapidity window. The $\langle\ldots\rangle$ denotes further below averaging over all events. So we introduce the correlation function

$$
\langle B\rangle_{F}=f(F)
$$


In principle one can study three main types of long-range correlations in line with the ALICE programme [四]:

$n-n$ - the correlation between values of multiplicities of charged particles in these two rapidity intervals,

$p_{t}-p_{t}$ - the correlation between values of the event mean transverse momenta in these intervals, and $p_{t}-n$ - the correlation between the values of mean transverse momenta in one rapidity interval and the multiplicity of charged particles in another interval. The present paper starts this program from the investigations of the correlation between multiplicities.

Forward-backward correlations have been characterized by the correlation strength, $b_{c o r}$ - the slope extracted from a measured - usually linear - relationship between the average multiplicity in some pseudorapidity interval in the backward hemisphere $\left(\left\langle n_{B}\right\rangle\right)$ and the multiplicity in the forward pseudorapidity window, $n_{F}$ [37]:

$$
<n_{B}>_{n_{F}}=a+b_{\text {cor }} \cdot n_{F}
$$

The linear relation of Eq.2.2 has been observed experimentally [B8],[37] and has also been discussed by Fowler et al.[B]].

The alternative definition of the correlation coefficient is possible through the direct calculation of the corresponding correlator[4]]:

$$
b_{c o r}=\frac{\left\langle n_{B} n_{F}\right\rangle-\left\langle n_{B}\right\rangle\left\langle n_{F}\right\rangle}{\left\langle n_{F}^{2}\right\rangle-\left\langle n_{F}\right\rangle^{2}} .
$$

It can be shown that both definitions are equal in case of linear correlation function. The correlation strength $b_{c o r}$ can be positive or negative with a range of $\left|b_{c o r}\right|<1$ (in case of equal rapidity intervals). This maximum (minimum) represents total correlation (anti-correlation) of the produced particles separated in rapidity. The $b_{c o r}=0$ corresponds to entirely uncorrelated particle production.

As it was mentioned above, one of the main features of the results obtained earlier for $n-n$ correlation is the linear dependence observed in the majority of cases for the correlation function. So, a linear parametrization Eq. 2.2 could be applied. It allows us to extract - by fitting - the coefficient $b_{c o r}$, the strength of the multiplicity correlation in the given data sample. This correlation coefficient $b_{\text {cor }}$ is a measure of the response of $\langle B\rangle_{F}$ on the variations of the variable $F$ in the vicinity of its average value $\langle F\rangle$.

To decrease the influence of some experimental factors like efficiency of registration and etc. and eliminate trivial dependence of the correlation coefficient on the width of the windows - in the case of nonequal forward and backward windows - it is useful to go to the relative (normalized) variables, i.e. to measure a deviation of $F$ from its average value $\langle F\rangle$ in units of $\langle F\rangle$, and the same for $B$. So we use in our analysis also the "relative" or "normalized" coefficient $b_{c o r}^{\text {rel }}$ for correlation between multiplicities $n_{B}$ and $n_{F}$ in backward and forward rapidity windows:

$$
\frac{\left\langle n_{B}\right\rangle_{n_{F}}}{\left\langle n_{B}\right\rangle}=a^{\text {rel }}+b_{c o r}^{\text {rel }} \frac{n_{F}-\left\langle n_{F}\right\rangle}{\left\langle n_{F}\right\rangle}
$$

The $n_{B}, n_{F}$ are the multiplicities of the charged particles, produced in the given event correspondingly in the backward $\left(\delta \eta_{B}\right)$ and forward $\left(\delta \eta_{F}\right)$ rapidity windows. 
It is clear that this "relative" coefficient is simply connected with "absolute" correlation coefficients defined above:

$$
b_{c o r}^{r e l}=\frac{\left\langle n_{F}\right\rangle}{\left\langle n_{B}\right\rangle} b_{c o r}
$$

and coincides with it in the case of symmetric windows.

The alternative approach of Eq.2.3 is also applied in our case of minimum bias $p p$ collisions data for the calculation of the multiplicity correlation coefficient. This allows us to crosscheck the results and to estimate a possible contribution to the systematic errors.

\section{Experimental Setup}

Studies of the multiplicity correlation coefficients were done at three values of $p p$ collision energies using the event-by-event charged-particle tracking data measured by the Inner Tracking System (ITS) and the Time Projection Chamber (TPC). Both detectors are located in the $0.5 \mathrm{~T}$ magnetic field.Charged particle tracks were measured within the pseudorapidity range $|\eta|<0.8$. A detailed general description of the ALICE experiment at the LHC can be found in [44]].

The ITS is composed of 3 different types of coordinate-sensitive Si-detectors and consists of 2 silicon pixel innermost layers, 2 silicon drift and 2 silicon strip outer detector layers. This design provides registration of high multiplicity events (with up to 100 charged particles per $\mathrm{cm}^{2}$ ). All ITS elements were carefully optimized to minimize their radiation length, achieving $1.1 \%$ Xo per layer - that is the lowest value among all the current LHC experiments. It provides a reliable charged particles tracking starting from $0.1 \mathrm{GeV} / \mathrm{c}$. Silicon drift and silicon strip detector layers have analogue readout and therefore can be used for particle identification via $d E / d x$ measurement in the non-relativistic region.

The ALICE TPC is the main tracking detector of the central rapidity region. It was designed to provide, together with the ITS and ALICE TRD and TOF, charged particle momentum measurement, particle identification and vertex determination with sufficient momentum resolution, two track separation and $d E / d x$ resolution for studies of hadronic and leptonic signals in the region of $p_{t}$ below $10 \mathrm{GeV} / c$. Good hadron identification by ALICE over a very broad momentum range, in particular in "soft" physics domain of $0.13 \mathrm{GeV} / \mathrm{c}$, is one of the most unique features of ALICE and a very strong point in its competitiveness with respect to the other two large LHC experiments.

\section{Data analysis}

The proton-proton collision data used in this analysis were collected by the ALICE experiment in 2010 and 2011. The data samples include $2 \mathrm{mln}$ events for $\sqrt{s}=0.9,10 \mathrm{mln}$ for $\sqrt{s}=2.76$ and $6.5 \mathrm{mln}$ for $\sqrt{s}=7 \mathrm{TeV}$ energy. The minimum bias trigger was used that required the crossing of two filled bunches and a signal in at least one of the two SPD pixel detector layers or in one of the additional multiplicity counters. The Event Selection was performed using the usual ALICE requirements of the vertex reconstruction and definite position.

For track selection some ALICE usual quality conditions were used that are defining acceptable track candidates. Besides this usual set, many other cut variations were also applied in selection criteria during the analysis of possible systematic effects in multiplicity measurements (see below). 


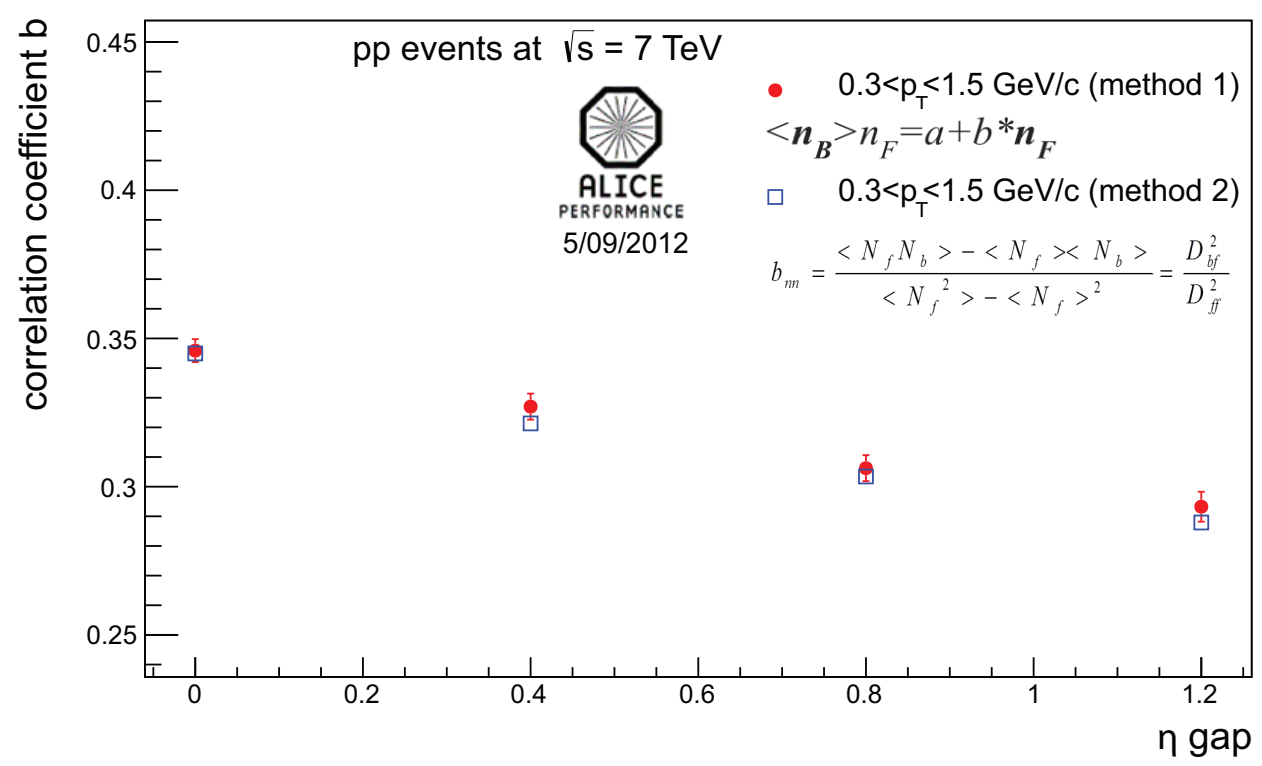

Figure 1: Two methods of calculation of forward-backward multiplicity correlation strength vs. $\eta$ gap (example)

\subsection{Experimental determination of the FB correlation coefficient (two methods)}

After been defined, charged particle tracks were required to belong to the selected pseudorapidity "forward" or "backward" windows $\delta \eta_{F}$ and $\delta \eta_{B}$.

Besides, the values of the $p_{t}$ of the track (i.e. of the transverse momentum of the particles in these rapidity intervals) were also required to be within the selected physics region of particle transverse momenta $\Delta p_{F}$ and $\Delta p_{B}$ :

$$
\eta_{i} \in \delta \eta \text { and }\left|\mathbf{p}_{t i}\right| \in \Delta p \text { for } i=1, \ldots, n
$$

The present work is focused on the search of the backward-forward correlation phenomenon in the production of "soft" hadrons with $p_{t}$ below $1.5 \mathrm{GeV} / \mathrm{c}$. Therefore we select in this study the tracks with $p_{t}$ within the region of $0.3 \mathrm{GeV} / c<p_{t}<1.5 \mathrm{GeV} / c$. This interval is also taken to ensure the efficiency plateau in $p_{t}$ for charged particle tracks registration in order to avoid the event-by-event corrections and to minimize the systematic errors at this stage of investigations. We have to note here that the $p_{t}$-dependent efficiency corrections, relevant to the detector performance, are difficult to apply to the event-by-event data(contrary to the studies of the global observables). This is because the origin of correlation is not known and, in case if the model correction is large, a strong bias of the experimental result will be inevitable.

Determination of the multiplicity forward-backward correlation coefficients was cross-checked by two methods: (i) using the linear fitting in relative variables (Eq.2.4) and (ii) using the alternative definition through the direct calculation of the corresponding correlator Eq. 2.3.

In the 1st method, the $\left(n_{B}, n_{F}\right) 2 \mathrm{D}$ distributions were accumulated event-by-event after the event and track selection using data in the given forward(backward) rapidity intervals. At the 


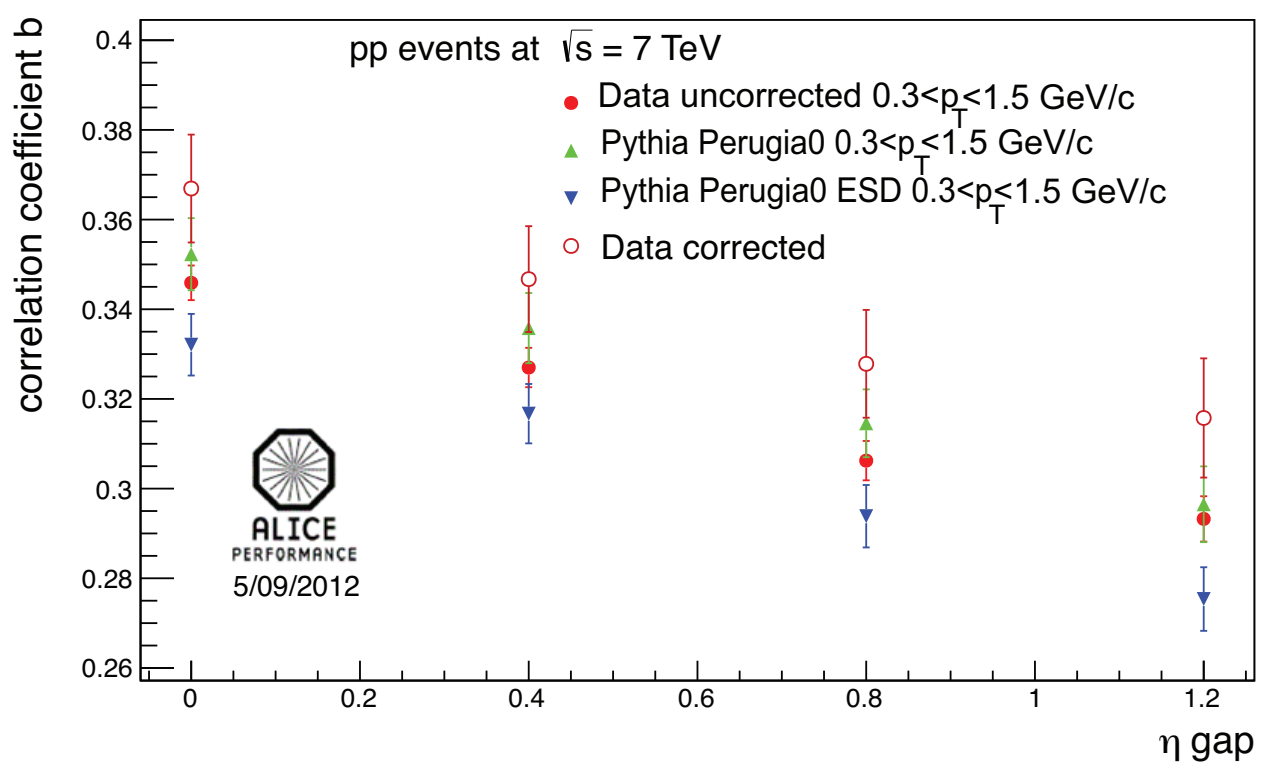

Figure 2: PYTHIA based corrections to the values of the multiplicity correlation strength (an example, see text). Note zoomed Y scale.

next step these raw data of 2D distributions were used for the determination of the average values of variables in the backward at the fixed values of some variable in the forward window. Then the relative (normalized) values were introduced (Eq.2.4) and the correlation coefficient $b_{\text {cor }}^{\text {rel }}$ was defined by fitting the experimental correlation function. In the 2 nd method the values of $\left\langle n_{B} n_{F}\right\rangle$, $\left\langle n_{B}\right\rangle,\left\langle n_{F}\right\rangle,\left\langle n_{F}^{2}\right\rangle$ and $\left\langle n_{F}\right\rangle^{2}$ were accumulated event-by-event and then the correlation coefficient was defined as indicated in Eq.2.3.

We have to mention that the definition Eq. .3 .3 does not enable to establish and to control the deviation (if it exists) of the correlation function ([2. لل from the linearity. However, these deviations were found at this stage of investigations of small statistical significance to influence the correlation coefficient value. The last statement is confirmed by a good agreement of these two methods applied, comparison of which is presented in the Fig.ll.

\subsection{Corrections and systematic error estimates (three procedures)}

Corrections to the measured multiplicity correlation coefficients were calculated by three independent procedures and results were compared. Some small discrepancies were included as a possible additional contribution to the final systematic error estimate.

The 1st procedure applied two types of the MC based (PYTHIA) calculations of $b_{c o r}^{\text {rel }}$ : with and without the experimental setup influence, producing so-called "measured" and "true" values of the correlation coefficients. The first case involved PYTHIA and the detailed GEANT model simulations of the ALICE installation where all factors that might influence the efficiency of track registration are taken into account. Thus the correction factors to the multiplicity correlation coefficients were defined (to each experimental point) as the relevant ratio of PYTHIA "true" and 


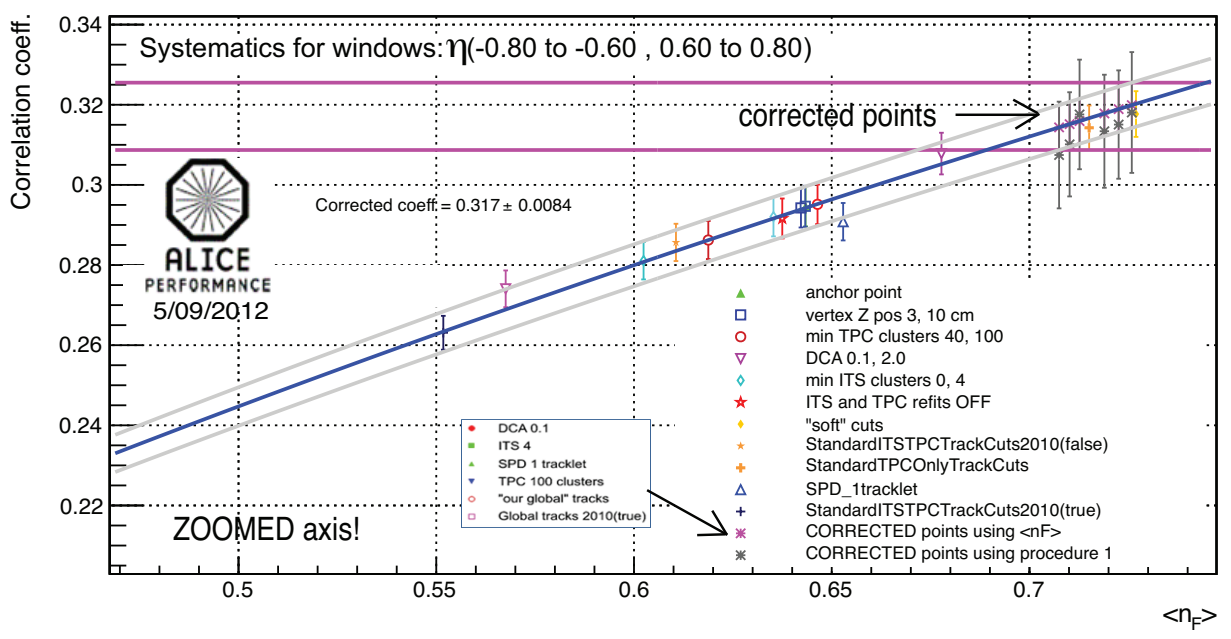

Figure 3: Multiplicity forward-backward correlation strength vs. $\left\langle N_{F}\right\rangle$ for various selection criteria and corrections (an example). Note zoomed Y scale.

"measured" values. One example of the experimental values of forward-backward multiplicity coefficients, measured in pp collisions at $7 \mathrm{TeV}$ as a function of the rapidity gap $\left(\eta_{\text {gap }}\right)$ between narrow $(\delta \eta=0.2)$ windows, is shown in Fig. 1 . The non-corrected points are marked by the black circles. Results by PYTHIA Perugia0 - with and without the experimental setup influence - are shown by the triangles. Corrected results are shown by the open circles.

The 2nd procedure was based on the direct correction of the inefficiencies in the measurements of values of multiplicity: $\left\langle n_{B} n_{F}\right\rangle,\left\langle n_{B}\right\rangle,\left\langle n_{F}\right\rangle,\left\langle n_{F}^{2}\right\rangle$ and $\left\langle n_{F}\right\rangle^{2}$. Efficiency factor was defined in each case as : "measured"/ "true" ratio. PYTHIA simulations - tuned to describe the mean multiplicity yields - were used to correct the multiplicities, then the corrected numbers were used in the corresponding correlator Eq. [2.3].

And the 3rd procedure took into account the influence of the various event and track selection parameters on the multiplicities. This influence was studied by the variations of selection criteria and comparison of $b_{c o r}^{r e l}$ to the value relevant to the standard ALICE sets of cuts. This procedure was also applied to correct each experimental data point. For each set of selection criteria the correlation coefficients with the relevant errors were determined for every windows pair in a way as it was described. It was obtained that these normalized multiplicity-multiplicity correlation coefficients are grouping along some narrow region and show some practically linear dependence on the corresponding "accumulated mean multiplicity" $n_{F}$ in the forward window (see Fig. [3). Thus, this procedure brings a possibility to extrapolate the value $b_{c o r}$ to the relevant "corrected" value $\left\langle n_{F}\right\rangle$. It is also possible to obtained both the corrected value of $b_{c o r}$ and the relevant corrected true multiplicity value. The example of the correction procedure applied for each point of the "accumulated mean multiplicity" value, and for each set of selection criteria mentioned, 


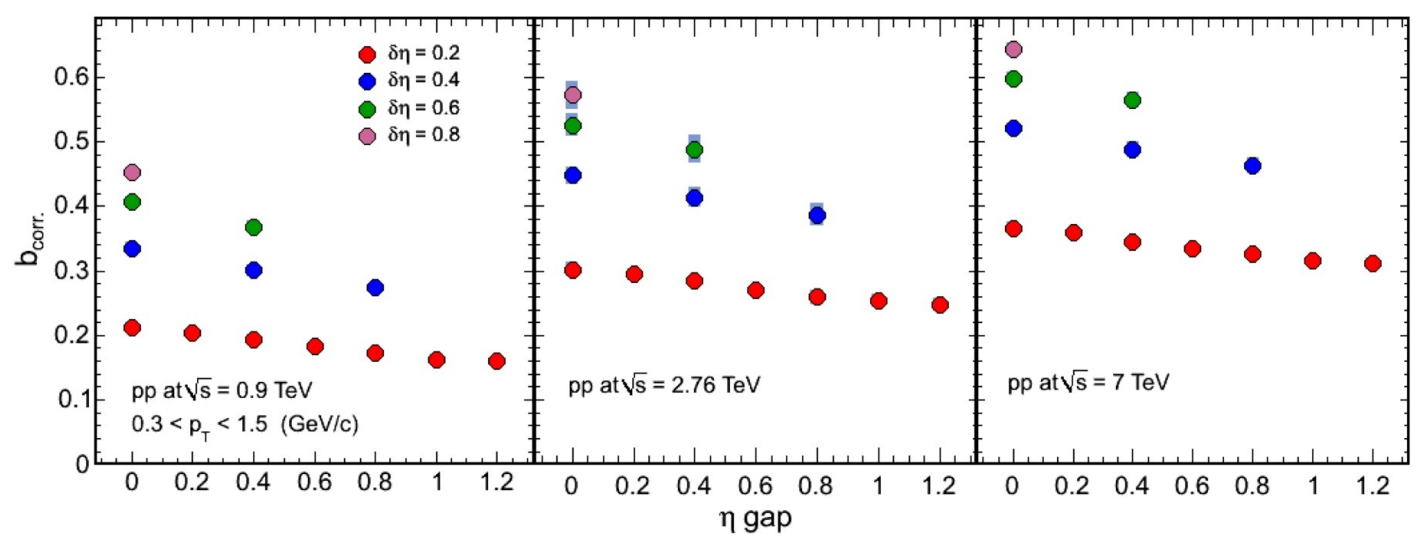

Figure 4: Multiplicity forward-backward correlation strength vs. $\eta$ gap and for different $\delta \eta$ bin-widths in pp collisions at $\operatorname{sqrt}(\mathrm{s})=0.9,2.76$ and $7 \mathrm{TeV}$. Results here and below are shown for the normalized observables.

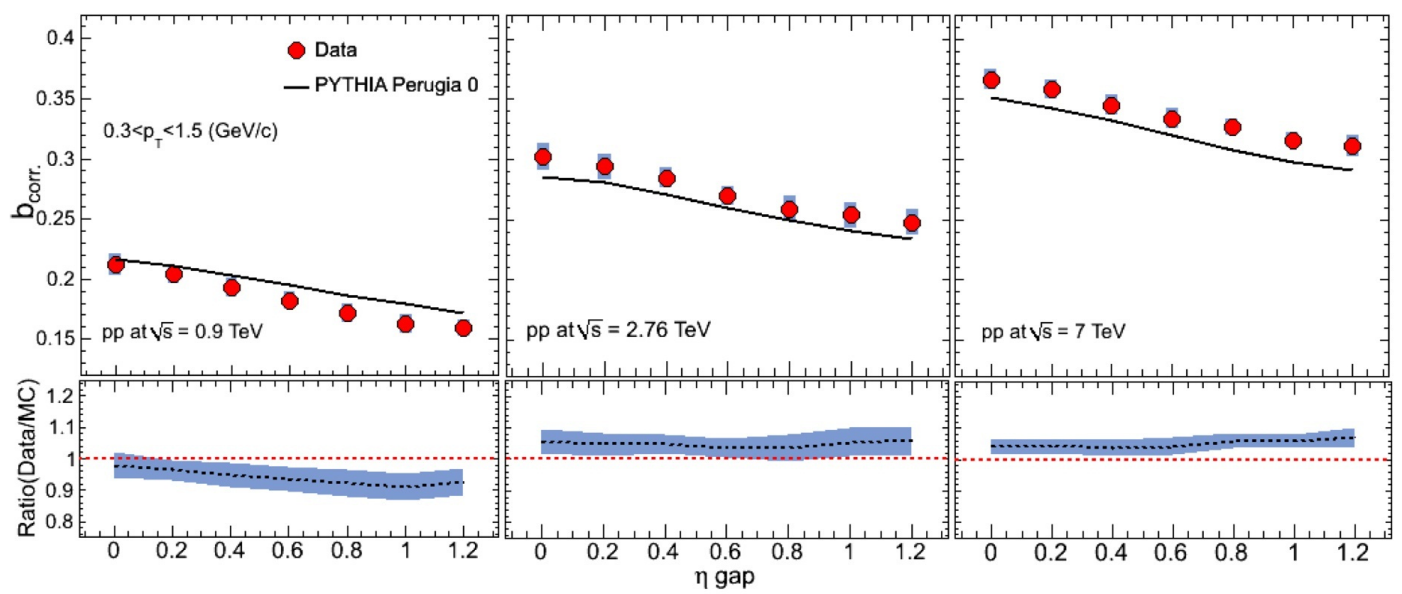

Figure 5: Forward-backward multiplicity correlation strength vs. $\eta$ gap and comparison to PYTHIA (Perugia 0 )for $\delta \eta=0.2$ in pp collisions at $\sqrt{s}=0.9,2.76$ and $7 \mathrm{TeV}$.

is shown in Fig. B]. The relevant spread of values of $b_{c o r}$ brings the major contribution to the systematic errors to the given point.

Various sources of systematic errors in multiplicity correlation strength measurements were studied. They including track selection criteria, methods of calculations, correction procedures, vertex determination errors, possible pile-up contribution. The final estimate gives the following upper limits of the total systematic errors of $5 \%, 4 \%$ and $3 \%$ at $0.9,2.76$ and $7 \mathrm{TeV}$ collision energies.

\section{Experimental results and discussion}

In Fig. $\$$ we show the final results for the coefficients of long-range $n$ - $n$ correlations vs. the $\eta$ gap value, obtained in the analysis of $p p$ collisions at $0.9,2.76$ and $7 \mathrm{TeV}$ for the normalized observables. 


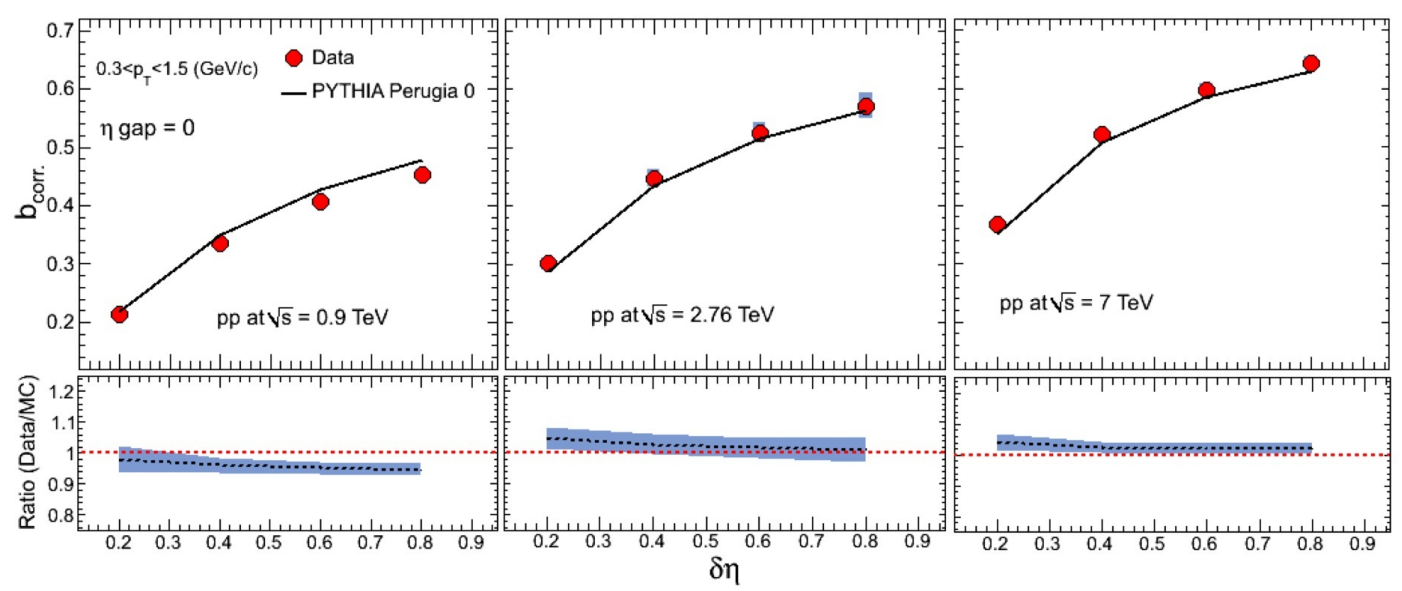

Figure 6: Forward-backward multiplicity correlation strength vs. the bin width $\delta \eta$ for $\eta_{g a p}=0$ in pp collisions at $\sqrt{s}=0.9,2.76$ and $7 \mathrm{TeV}$ and comparison to PYTHIA Perugia 0.

First of all, we see noticeable multiplicity correlations in all cases. The forward-backward multiplicity correlation strength is decreasing slowly with the growing $\eta$ gap between the windows. At the largest $\eta$ gap value, reached in this study ( $\eta$ gap=1.2), these correlations are losing only about $10 \%$ of strength. The PYTHIA(Perugia0) results are found to be in a qualitative agreement with the existence of non-negligible multiplicity correlations in the whole pseudorapidity region of studies (some examples of PYTHIA(Perugia0) calculations of $\eta_{\text {gap }}$ dependence, done for 3 LHC energies, are shown in Fig. (】).

Secondly, it is necessary to note that $b_{c o r}^{r e l}$, the strength of these correlations in normalized observables, is increasing - in a non-linear way - with $\delta \eta$ - the width of the observation "forward" window). Summary of data for $b_{c o r}^{r e l}$ vs. the different widths $\delta \eta$ of the observation windows are also shown separately in Fig. 6. One may see a tendency for saturation with the width of the "forward" window. PYTHIA is also showing the same non-linear behavior of the correlation strength vs. the width of the forward pseudorapidity window, presented in Fig.6. We have to note here that the multiplicity correlation strength (obtained in normalized observables) depends in such way only on the width of the forward pseudorapidity window, and it is practically constant with the variation of the width of the backward one.

We see that the correlation strength from PYTHIA(Perugia0) are in qualitative agreement with the available experimental data ( PYTHIA is working reasonably well in case of data of Fig.】 and 6 description). However PYTHIA(Perugia0) fails to describe the ratio of correlation strength $b$ at $7 \mathrm{TeV}$ and $2.76 \mathrm{TeV}$ wrt $0.9 \mathrm{TeV}$ vs. $\eta_{\text {gap }}$, see Fig.Ш. The last one shows the wrong energy dependence produced by PYTHIA. Recently the available data on forward-backward correlation strength as a function of pseudorapidity intervals were reviewed and compared to PYTHIA and PHOJET model calculations[45]]. It was obtained that PYTHIA is in a general better agreement with the data than PHOJET. However our new experimental data on multiplicity correlation strength, obtained at the LHC energies, are imposing new constraints on the theoretical descriptions.

Finally, one has to stress the fact that the general growth of the multiplicity correlation coefficient $b_{c o r}$ is observed in our study with the increase of $p p$ collision energy from 0.9 to $7 \mathrm{TeV}$. 


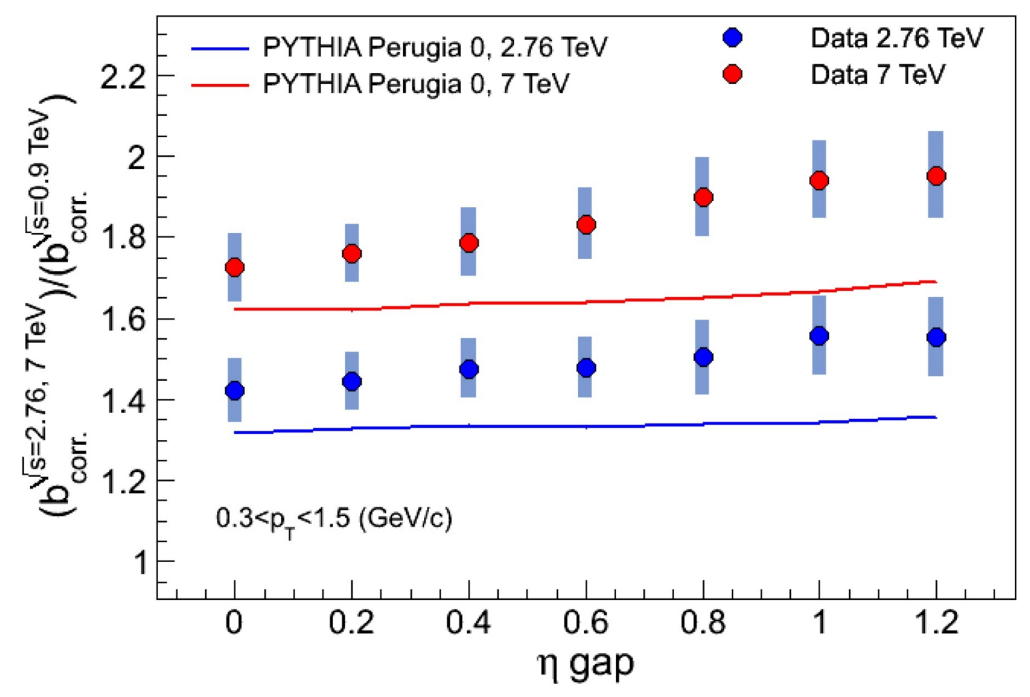

Figure 7: Ratio of correlation strength $b$ at $7 \mathrm{TeV}$ and $2.76 \mathrm{TeV}$ wrt $0.9 \mathrm{TeV}$ vs. $\eta$ gap.

It is remarkable to note that this growth of $b_{c o r}$ practically does not affect the slopes of $b_{c o r}$ weak dependencies on the $\eta$ gap (see Fig.(A). One may conclude that the short-range correlations contribution has a very weak dependence on the collision energy, while the long range multiplicity correlations plays a dominant role in pp collisions and its strength significantly increases with the collision energy growth from $900 \mathrm{GeV}$ to $7 \mathrm{TeV}$.

One may see that the observed relative decrease of the $b_{c o r}$ with the $\eta$ gap (see in Fig.(I) is practically not changing with the collision energy. PYTHIA results are confirming the general trend compatible with the LRC phenomena for the pp collisions. All these facts are pointing to the limited role of short range correlations here and are stressing the importance of variable pseudorapidity intervals in detailed studies of short- and long-range correlation effects. More of the experimental evidence will be provided by the ongoing studies by ALICE of the topology of long-range correlations for various observables measured in separated pseudorapidity ( $\delta \phi 0$ and azimuthal $(\delta \eta)$ intervals.

\section{Conclusion}

The forward-backward multiplicity correlations are experimentally studied in $p p$ collisions at $0.9 \mathrm{TeV}, 2.76$ and $7 \mathrm{TeV}$ with the $\eta_{\text {gap }}$ between pseudorapidity windows extended up to 1.2 units. Strong non-linear dependence on the width of the pseudorapidity windows is demonstrated by the forward-backward multiplicity correlation strength for the normalized observables.

The general growth of the Forward-Backward multiplicity correlations strength with energy is obtained in pp collisions study in ALICE at 0.9, 2.76 and $7 \mathrm{TeV}$ for all widths of pseudorapidity forward windows. At the same time the experimental data show stable behavior (practically the same quantitative decrease) of the correlation coefficient with the growth of the pseudorapidity gap between forward and backward windows at all three collision energies. One may conclude that short-range correlations contribution has a very weak dependence on the collision energy, while 
the long range multiplicity correlation plays a dominant role in $p p$ collisions at the LHC and its strength significantly increases with the collision energy growth from $900 \mathrm{GeV}$ to $7 \mathrm{TeV}$.

The first comparison with the PYTHIA Perugia- 0 calculations on the dependence of the correlation strength on the collision energy, the width and the position of pseudorapidity windows show that the experimental data of the present study impose new constraints on the theoretical models.

Acknowledgement One of the authors (V.V.) is partially supported by the RFFI grant 12-0200356-a.

\section{References}

[1] ALICE collaboration. "ALICE: Physics Performance Report, Volume II" J. Phys. G32 1295-2040 (Section: 6.5.15 - Long-range correlations, p.1749-1751), (2006) .

[2] A.Dumitru et al./ Nuclear Physics A 810, 91-108(2008).

[3] N. Armesto, L. McLerran, and Pajares C. Nucl. Phys., A781-201, (2007).

[4] K.Wraight, P.Skands, ArXiv: 1101,5215v1,[hep-ph], 27Jan (2011).

[5] W. Braunschweig et al. (TASSO Collaboration). Z. Phys., C45-193, (1989).

[6] P. Abreu et al. (DELPHI Collaboration). Z. Phys., C50-185, (1991).

[7] R. Akers. Phys. Lett., B320-417, (1994).

[8] C.M. Bromberg et al. Phys. Rev., D9-1864, (1974).

[9] S. Uhlig et al. Nucl. Phys., B132-15, (1978).

[10] R.E. Ansorge et al. (UA5 Collaboration). Z. Phys., C37-191, (1988).

[11] T. Alexopoulos et al. Phys. Lett., B353-155, (1995).

[12] M. Arneodo et al. (European Muon Collaboration). Nucl. Phys., B258-249, (1985).

[13] V.V. Aivazyan et al. (NA22 Collaboration). Z. Phys., C42-533, (1989).

[14] W.D. Walker. Phys. Rev., D69-034007-1, (2004).

[15] T. Alexopoulos et al. (E735 Collaboration). Phys. Lett., B353-155, (1995).

[16] NA49 collaboration and Feofilov G.A., Kolevatov R.S.,Kondratiev V.P., Naumenko P.A., Vechernin V.V.,"Long-Range Correlations in PbPb Collisions at 158 AGeV", Proc. XVII ISHEPP, vol.1, 222-231, JINR, Dubna, (2005).

[17] A.Asryan, G.Feofilov, A.Ivanov, A.Grebenyuk, P.Naumenko and V.Vechernin (for ALICE Collaboration), "Long-Range Multiplicity Correlation in pp-collisions in ALICE at the LHC", Proc. ISHEPP-XIX,vol.2, pp.208-214m JINR, Dubna, (2008).

[18] B.I. Abelev (STAR Collaboration). arXiv-nucl-ex/0905.0237, (2009).

[19] T.J. Tarnowsky. Ph.d. thesis, purdue university. arXiv-nucl-ex/0807.1941, (2008).

[20] V.P. Konchakovski et al. Phys. Rev., C79-034910, (2009).

[21] P. Brogueira, J. Dias de Deus, and Pajares C. arXiv-hep-ph/0901.0997, (2009).

[22] A. Bzdak. arXiv-hep-ph/0902.2639, (2009).

[23] Yu-Liang Yan et al. arXiv-nucl-th/0903.0915, (2009). 
[24] M.A. Braun, C. Pajares, V.V. Vechernin, Phys. Lett. B493, 54 (2000).

[25] V.V. Vechernin, R.S. Kolevatov, hep-ph/0304295; hep-ph/0305136 (2003); Vestnik SPbU, ser.4, no.2, 12 (2004); ibid. no.4, 28 (2004).

[26] M.A. Braun, R.S. Kolevatov, C. Pajares, V.V. Vechernin, Eur. Phys. J. C32, 535 (2004).

[27] V.V. Vechernin, R.S. Kolevatov, Physics of Atomic Nuclei 70, 1797 (2007); ibid. 70, 1809 (2007).

[28] V.V. Vechernin, H.S. Nguyen, Phys. Rev. C 84, 054909 (2011).

[29] V.V. Vechernin, Proceedings of the Baldin ISHEPP XX, vol.2,10-15, JINR, Dubna, (2011); arXiv:1012.0214 [hep-ph] (2010).

[30] V.V. Vechernin, arXiv:1210.7588 [hep-ph] (2012).

[31] A.Capella, U.P.Sukhatme, C.-I.Tan and J.Tran Thanh Van, Phys. Lett. B81 68(1979); Phys. Rep.,236, 225,(1994). A.B. Kaidalov, K.A. Ter-Martirosyan, Phys.Lett. 117B, 247 (1982).

[32] Abramovskii V. A., Gedalin E. V., Gurvich E. G., Kancheli O. V., JETP Lett., vol.47, 337-339, (1988).

[33] M.A.Braun and C.Pajares, Phys. Lett. B287 (1992) 154; Nucl. Phys. B390 542, 549,(1993);

[34] N.S.Amelin et al. Phys. Rev. Lett. 73, 813 (1994).

[35] L.McLerran, Nucl.Phys.A699,73c(2002).

[36] T. Lappi, L. McLerran, arXiv:0909.0428, (2009); Nucl. Phys. A832, 330 (2010).

[37] UA5 Collaboration, Z.Phys,C-Particles and Fields 37,191-213 (1988)

[38] S. Uhlig et al., Nucl. Phys. B 132 , 15, (1978).

[39] G.N. Fowler et al., Phys. Rev. D 37, 3127, (1988).

[40] A. Capella and J. Tran Thanh van. Phys. Rev., D29-2512, (1984).

[41] A.Capella et al.,Phys.Rep. 236,225(1994).

[42] A. Capella and A. Krzywicki. Phys. Rev., D18-4120, (1978).

[43] A. Capella and J. Tran Thanh van. Z. Phys., C18-85, (1983).

[44] ALICE Collaboration, J. Instrum. 3, S08002 (2008).

[45] A.K.Dash, D.P. Mahapatra,B.Mohanty, arXiv:1204.5017v1, [hep=ex] 23 April 2012. 